Tropical Journal of Pharmaceutical Research December 2015; 14 (12): 2213-2216

ISSN: $1596-5996$ (print); 1596-9827 (electronic)

(c) Pharmacotherapy Group, Faculty of Pharmacy, University of Benin, Benin City, 300001 Nigeria.

All rights reserved.

Available online at http://www.tjpr.org

Original Research Article

http://dx.doi.org/10.4314/tjpr.v14i12.9

\title{
Differences in Expression of EGFR, Ki67 and p-EPK in Oral Cavity Squamous Cell Carcinoma
}

\author{
Jing-qiu Bu ${ }^{1 *}$, Xi Bu ${ }^{2}$, Peng Chen ${ }^{1}$, Bing Liu ${ }^{3}$ and Fei Chen ${ }^{1}$ \\ ${ }^{1}$ Department of Stomatology, Chinese People's Liberation Army General Hospital, No.28 Fuxing Road, Haidian District, Beijing \\ 100853, ${ }^{2} 5$ Years of Clinical Medicine 97, the New Campus of China Medical University, No. 77 Puhe Road, Shenyang, \\ Liaoning Province 110013, ${ }^{3}$ Department of Stomatology, the General Hospital of the Air Force of the Chinese People's \\ Liberation Army, No. 30 Fucheng Road, Haidian District, Beijing 100142, China
}

*For correspondence: Email: JingqiuBu133494@163.com; Tel: +86 010-66938216

Received: 5 November 2015

Revised accepted: 25 September 2015

\begin{abstract}
Purpose: To evaluate the expression of EGFR, Ki67, and p-EPK in oral cavity and oropharyngeal cancers, and to investigate their clinical significance as prognostic markers.

Methods: One hundred patients who underwent curative surgery for oral cavity or oropharyngeal squamous cell carcinoma in a Chinese People's Liberation Army General Hospital between March 1999 and October 2010 were evaluated. The level of protein expression of EGFR, Ki67 and p-EPK was assessed by immunohistochemistry. In situ hybridization was used to detect the existence of human papillomavirus (HPV).

Results: Nineteen of 75 patients with oropharyngeal cancer showed HPV-positive tumors, and two of 72 patients with oral cavity cancer showed HPV-positive tumors. EGFR and Ki67 expression was significantly higher in oral cavity cancers than in oropharyngeal cancers $(p=0.005$ and $p=0.001$, respectively). Loss of $p$-EPK occurred significantly more frequently in oral cavity cancers than in oropharyngeal cancers $(p=0.004)$. Overexpression of EGFR and Ki67 and loss of $p-E P K$ were observed more frequently in HPV-negative tumors. Multivariate Cox regression analysis showed that Ki67 expression had a significantly unfavorable impact on relapse-free survival in oropharyngeal cancer. Conclusion: The expression levels of EGFR, Ki67, and p-EPK differ between oropharyngeal and oral cavity cancer and it may be attributed to HPV-related molecular pathogenesis. The expression of Ki67 might be an unfavorable prognostic marker for relapse-free survival in oropharyngeal cancer.
\end{abstract}

Keywords: EGFR; Ki67; p-EPK; Oral cavity squamous cell carcinoma, Expression difference

Tropical Journal of Pharmaceutical Research is indexed by Science Citation Index (SciSearch), Scopus, International Pharmaceutical Abstract, Chemical Abstracts, Embase, Index Copernicus, EBSCO, African Index Medicus, JournalSeek, Journal Citation Reports/Science Edition, Directory of Open Access Journals (DOAJ), African Journal Online, Bioline International, Open-J-Gate and Pharmacy Abstracts

\section{INTRODUCTION}

Mucosal squamous cell carcinoma of the head and neck (SCCHN) comprises a heterogeneous group of tumors arising from the epithelial lining of the oral cavity, pharynx, and larynx. Despite distinct clinical characteristics, oral cavity cancer (OSCC) and oropharyngeal cancer (OPSCC) are frequently described as part of a group of oral cancers, and the two disease entities are often confused in the literature. OSCC includes the lips, buccal mucosa, teeth, gums, anterior twothirds of the tongue, floor of the mouth and hard palate. OPSCC includes the base of the tongue, soft palate, tonsils, and the lateral and posterior pharyngeal walls.

In recent decades, studies have shown that OSCC and OPSCC have different incidence rate trends, etiology, and survival outcome [1]. For instance, human papillomavirus (HPV) is known as an important etiology for OPSCC, especially 
tonsil cancer. However, there is little research on the differences in molecular pathogenesis between the two diseases. Aberrant epidermal growth factor receptor (EGFR) affects cell cycle progression, apoptosis, angiogenesis, and metastasis. EGFR is overexpressed in about 90 $\%$ of all SCCHNs, and its overexpression correlates with poor prognosis [2]. The phosphatidylinositol 3-kinase (PI3K)/Akt pathway is important for cell survival because it promotes cell cycle progression and inhibits apoptosis [3]. Mammalian target of rapamycin (mTOR), a serine/threonine kinase and a downstream target of Akt, is important for the oncogenic transformation induced by $\mathrm{PI} K \mathrm{~K}$ and Akt. Signaling through the PI3K/Akt/mTOR pathway is activated by growth factor ligand binding to receptor tyrosine kinases and regulates several cellular functions that are critical for tumorigenesis. The $\mathrm{p}-\mathrm{EPK}$, a tumor suppressor gene, negatively regulates the PI3K/Akt pathway. The loss of $p$-EPK function causes increased Ki67 activity and continued cell survival and proliferation [4]. In this study, we evaluated the expression of EGFR, Ki67 and p-EPK in OSCC and OPSCC, and investigated their clinical significance as a prognostic marker.

\section{EXPERIMENTAL}

\section{Patients}

One hundred patients who underwent curative surgery for OPSCC or OSCC in Chinese People's Liberation Army General Hospital between March 1999 and October 2010 were evaluated. Among the 100 patients, 51 (51.0\%) had been diagnosed with OPSCC and 49 (49.0 $\%)$ with OSCC. Clinical records and pathology reports were reviewed retrospectively. The following clinical data were collected: age, sex, smoking history, tumor staging, surgery type, chemotherapy, radiotherapy, recurrence, and survival. Ethical committee approval (ref no. 201406005) was obtained from the Institutional Review Board of Beijing University. Informed consent was provided according to the Declaration of Helsinki.

\section{In situ hybridization for HPV}

In situ hybridization was processed on the automated Benchmark system from Ventana Medical Systems using INFORM HPV III Family 16 Probe (cocktail of HPV subtypes 16, 18, 31, 33, 35, 39, 45, 51, 52, 56, and 66, Ventana Medical Systems) as per the manufacturer's recommendations. This system removes the paraffin wax from the tissue, subjects it to protease digestion, and then hybridizes the tissue with a probe. The probe-target complex is detected because of the action of alkaline phosphatase on chromogen nitroblue tetrazolium and bromochloroindolyl phosphate, which yields a dark-blue color with a pink counterstain for the HPV-negative cells caused by nuclear fast red staining. The signal patterns of HPV in the nuclei were classified as follows: diffuse, signals that were condensed and uniformly packed in the nucleus; punctate, signals that were dot-like and distributed sparsely in the nucleus.

\section{Statistical analysis}

Overall survival (OS) was defined as the time from the date of diagnosis to the date of death or last follow-up. Relapse-free survival (RFS) was defined as the time from surgery to disease recurrence. Continuous and categorical variables were compared using Student's t test and the chi-square test. Univariate analysis and survival curves were estimated using the Kaplan-Meier method, and the log-rank test was applied to identify differences. Multivariate analysis was performed with Cox hazards regression model. All statistical analyses were performed using SPSS program (version 18.0) and $p<0.05$ was considered significant.

\section{RESULTS}

\section{Patient characteristics}

The clinicopathological characteristics of the 100 patients are summarized in Table 1. The mean age of the patients was 52.8 (range $25-90$ ) years. Among the 51 OPSCC patients, 48 (78.7 $\%)$ patients had tonsil cancer, $9(14.7 \%)$ patients had cancer of the base of the tongue, $2(3.3 \%)$ patients had soft palate cancer, and 2 (3.3\%) patients had pharyngeal wall cancer. Among the 49 OSCC patients, $51(85.0 \%)$ patients had tongue cancer, $5(8.3 \%)$ patients had buccal mucosa cancer, and $4(6.7 \%)$ patients had cancer of the floor of the mouth. The percentage of men was higher in those with OPSCC (93.4 $\%)$ than in those with OSCC $(63.3 \%, p=0.001)$. $19(31.1 \%)$ of 61 patients with OPSCC showed HPV-positive tumors, and only $2(3.3 \%)$ of 60 patients with OSCC showed HPV-positive tumors $(p=0.001)$. Well-differentiated histological type was more frequent in OSCC than in OPSCC $(p=$ 0.001 ). Cervical lymph node metastasis with advanced $\mathrm{N}$ stage was more frequent in OPSCC than in OSCC $(p=0.002)$. Adjuvant therapy after surgery was followed more frequently in OPSCC than in OSCC $(p=0.031)$. Age, smoking history, and $T$ stage did not differ significantly between patients with OPSCC and OSCC. 


\section{Immunohistochemical staining and correlation analysis}

The results of immunohistochemical staining are listed in Table 2. EGFR and Ki67 expression was significantly higher in OSCC than in OPSCC $(p=$ 0.005 and $p=0.001$, respectively). Loss of $p$ EPK was more frequent in OSCC than in OPSCC $(p=0.004)$. Overexpression of EGFR and Ki67 was observed more frequently in HPVnegative tumors than in HPV-positive tumors ( $p=$ 0.003 and $p=0.037$, respectively). Loss of $p$ -
EPK was observed more frequently in HPVnegative tumors than in HPV-positive tumors $(p=$ 0.001 ). Ki67 overexpression and loss of $p$-EPK occurred more frequently in tumors with welldifferentiated histology $(p=0.003$ and $p=0.006$, respectively). We analyzed the individual relationships between EGFR, Ki67 and p-EPK expression. p-EPK and Ki67 expression correlated inversely $(p=0.032)$. Other variables did not correlate significantly.

Table 1: Clinicopathological characteristics of oropharyngeal and oral cavity cancer patients

\begin{tabular}{|c|c|c|c|c|}
\hline Variable & $\begin{array}{c}\text { All patients } \\
(\mathrm{n}=100) \\
\text { No. }(\%)\end{array}$ & $\begin{array}{l}\text { OPSCC } \\
(n=51) \\
\text { No. }(\%)\end{array}$ & $\begin{array}{c}\text { OSCC } \\
(n=49) \\
\text { No. }(\%)\end{array}$ & $p$-value \\
\hline \multicolumn{5}{|l|}{ Age (years) } \\
\hline MeanıSD & $52.8 \pm 9.4$ & $53.5 \pm 8.7$ & $52.4 \pm 11.2$ & 0.608 \\
\hline \multicolumn{4}{|l|}{ Sex } & 0.001 \\
\hline Men & $72(72.0)$ & $48(94.1)$ & $36(73.5)$ & \\
\hline Women & $28(28.0)$ & $3(5.9)$ & $13(26.5)$ & \\
\hline \multicolumn{4}{|l|}{ Smoking } & 0.672 \\
\hline Former or current & $71(71.0)$ & $32(62.7)$ & $32(65.3)$ & \\
\hline \multirow{2}{*}{\multicolumn{4}{|c|}{ HPV status }} & \\
\hline & & & & 0.001 \\
\hline Positive & $25(25.0)$ & $16(31.4)$ & $4(8.3)$ & \\
\hline Negative & $75(75.0)$ & $35(68.6)$ & $45(91.8)$ & \\
\hline \multicolumn{4}{|l|}{ Histologic grade } & 0.001 \\
\hline Well & $31(31.0)$ & $10(20.0)$ & $28(57.1)$ & \\
\hline Moderate & $56(56.0)$ & $32(62.7)$ & $15(30.6)$ & \\
\hline Poor & $13(13.0)$ & $8(17.3)$ & $7(14.3)$ & \\
\hline \multicolumn{4}{|l|}{ T stage } & 0.057 \\
\hline$<2 \mathrm{~cm}$ & $32(32.0)$ & $10(20)$ & $21(42.9)$ & \\
\hline$\gg 2 \mathrm{~cm},<4 \mathrm{~cm}$ & $59(59.0)$ & $33(64.7)$ & $24(49.0)$ & \\
\hline$\gg 4 \mathrm{~cm}$ & $9(9.0)$ & $7(15.3)$ & $5(10.2)$ & \\
\hline \multicolumn{4}{|l|}{ N stage } & 0.003 \\
\hline No & $51(51.0)$ & $9(17.6)$ & $27(55.1)$ & \\
\hline N1 & $13(13.0)$ & $8(15.7)$ & $8(16.3)$ & \\
\hline $\mathrm{N} 2 \mathrm{a}$ & $3(3.0)$ & $3(5.9)$ & $2(4.1)$ & \\
\hline $\mathrm{N} 2 \mathrm{~b}$ & $27(27.0)$ & $14(27.5)$ & $9(18.4)$ & \\
\hline N2c & $6(6.0)$ & $16(31.4)$ & $4(8.2)$ & \\
\hline \multicolumn{5}{|l|}{ Treatment } \\
\hline Surgery alone & $36(36.0)$ & $14(27.5)$ & $24(49.0)$ & 0.028 \\
\hline Adj RT & $31(31.0)$ & $21(41.2)$ & $14(28.6)$ & 0.079 \\
\hline Adj CCRT & $22(22.0)$ & $10(19.6)$ & $7(14.3)$ & 0.459 \\
\hline Adj CTx & $11(11.0)$ & $5(9.8)$ & $5(10.2)$ & 0.321 \\
\hline
\end{tabular}

Table 2: Immunohistochemical staining for EGFR, Ki67 and p-EPK

\begin{tabular}{|c|c|c|c|c|}
\hline Variable & $\begin{array}{c}\text { All patients } \\
(\mathrm{n}=100) \\
\text { No. }(\%)\end{array}$ & $\begin{array}{l}\text { OPSCC } \\
(n=51) \\
\text { No. }(\%) \\
\end{array}$ & $\begin{array}{c}\text { OSCC }(n=49) \\
\text { No. }(\%)\end{array}$ & $p$-value \\
\hline EGFR & & & & 0.006 \\
\hline Positive & $63(63.0)$ & $28(54.9)$ & $31(63.3)$ & \\
\hline Negative & 37(37.0) & $23(45.1)$ & $18(36.7)$ & \\
\hline Ki67 & & & & 0.004 \\
\hline Positive & $12(8.9)$ & $21(17.9)$ & $25(26.6)$ & \\
\hline Negative & $85(91.1)$ & $18(56.7)$ & $22(47.5)$ & \\
\hline$p-E P K$ & & & & 0.201 \\
\hline Positive & $64(65.6)$ & $32(74.2)$ & $24(47.2)$ & \\
\hline Negative & $29(34.4)$ & 18(20.8) & 17(31.6) & \\
\hline
\end{tabular}




\section{DISCUSSION}

Carcinomas of the oropharynx and oral cavity constitute $2-5 \%$ of SCCHNs. In the past, OPSCC and OSCC were considered types of oral cancers with the same etiology including smoking and alcohol abuse. However, there is growing evidence to support the idea that OPSCC and OSCC are two distinct diseases with different etiologies [5].

Since the 1980s, evidence has been emerging that HPV is an etiological factor for a subset of OPSCC [6]. Previous studies reported that the rate of HPV positivity is higher in OPSCC, especially tonsil cancer, than in OSCC [7]. Our result is consistent with these previous results: about $30 \%$ of OPSCC patients showed HPV positivity, in contrast to $3 \%$ of OSCC patients.

We compared the expression of the EGFR and $\mathrm{Ki67/p-EPK} \mathrm{pathway,} \mathrm{which} \mathrm{is} \mathrm{crucial} \mathrm{for} \mathrm{cell}$ survival and growth, in OPSCC and OSCC patients. EGFR and Ki67 expression was significantly higher in OSCC than in OPSCC, and loss of $p$-EPK occurred more frequently in OSCC than in OPSCC.

The correlation analysis between the expression of these proteins and HPV status showed that overexpression of EGFR and Ki67 and loss of pEPK were observed more frequently in HPVnegative tumors. Therefore, these differences in EGFR, Ki67 and p-EPK expression between OPSCC and OSCC may be attributed to HPVrelated molecular pathogenesis.

Previous studies showed that Ki67 expression is associated with poor outcome in OPSCC and OSCC. Massarelli et al reported that tongue cancer patients with Ki67 expression showed significantly shorter disease-free survival [8]. Yu et al reported that OPSCC patients with a low Ki67 level had a lower 5-year local recurrence rate and better 5-year overall survival rate [9].

However, research on the relationship between Ki67 expression and HPV status is limited. In the present study, Ki67 overexpression was significantly lower in HPV-positive tumors than in HPV-negative tumors. Analysis of RFS showed that $\mathrm{T}$ and $\mathrm{N}$ stages were only meaningful as a prognostic factor in OSCC patients. Meanwhile, OPSCC patients with Ki67 overexpression tended to have a lower 3-year RFS rate, and in patients with tonsil cancer, the overexpression of Ki67 was associated with significantly shorter RFS.

\section{CONCLUSION}

The findings of this study reveal that Ki67 may be a prognostic marker for RFS in OPSCC patients, especially in those with tonsil cancer. Further studies are needed to identify more clearly the role of EGFR and Ki67/p-EPK pathway in HPVrelated OPSCC.

\section{ACKNOWLEDGEMENT}

This project was supported by Natural Science Foundation of Beijing City (no. 7122161).

\section{REFERENCES}

1. Auluck A, Hislop G, Bajdik C, Poh C, Zhang L, Rosin M. Trends in oropharyngeal and oral cavity cancer incidence of human papillomavirus (HPV)-related and HPV-unrelated sites in a multicultural population: the British Columbia experience. Cancer 2010; 116: 2635-2644.

2. Machiels JP, Schmitz S. Molecular-targeted therapy of head and neck squamous cell carcinoma: beyond cetuximab-based therapy. Curr Opin Oncol 2011; 23: 241-248.

3. Morgensztern D, McLeod HL. PIJK/Akt/mTOR pathway as a target for cancer therapy. Anticancer Drugs 2005; 16: 797-803.

4. Nagata $Y$, Lan $K H$, Zhou $X$, Tan M, Esteva FJ, Sahin AA. $P T E N$ activation contributes to tumor inhibition by trastuzumab, and loss of PTEN predicts trastuzumab resistance in patients. Cancer Cell 2004; 6: 117-127.

5. Marur S, Forastiere AA. Head and neck cancer: changing epidemiology, diagnosis, and treatment. Mayo Clin Proc. 2008; 83: 489-501.

6. Kim L, King T, Agulnik M. Head and neck cancer: changing epidemiology and public health implications. Oncology (Williston Park) 2010; 24: 915-919.

7. Laco J, Vosmikova $H$, Novakova V, Celakovsky $P$, Dolezalova $H$, Tucek $L$. The role of high-risk human papillomavirus infection in oral and oropharyngeal squamous cell carcinoma in non-smoking and nondrinking patients: a clinicopathological and molecular study of 46 cases. Virchows Arch 2011; 458: 179187.

8. Massarelli E, Liu DD, Lee JJ, El-Naggar AK, Lo Muzio L, Staibano S. Akt activation correlates with adverse outcome in tongue cancer. Cancer 2005; 104: 24302436.

9. Yu Z, Weinberger PM, Sasaki C, Egleston BL, Speier WFT, Haffty B. Phosphorylation of Akt (Ser473) predicts poor clinical outcome in oropharyngeal squamous cell cancer. Cancer Epidemiol Biomarkers Prev. 2007; 16: 553-558. 